\title{
Reporting quality of surgical randomised controlled trials in head and neck cancer: a systematic review
}

\author{
Netanya Aarabi Canagarajah ${ }^{1} \cdot$ George James Porter $^{1} \cdot$ Kurchi Mitra $^{1} \cdot$ Timothy Shun Man Chu $^{1} \mathbb{C}$
}

Received: 15 November 2020 / Accepted: 9 February 2021 / Published online: 19 February 2021

(c) The Author(s) 2021

\begin{abstract}
Purpose Randomised controlled trials (RCTs) are considered the gold standard for evaluating the efficacy of an intervention. However, previous research has shown that RCTs in several surgical specialities are poorly reported, making it difficult to ascertain if various biases have been appropriately minimised. This systematic review assesses the reporting quality of surgical head and neck cancer RCTs.

Methods A literature search of PubMed and Embase was performed. Papers were included if they reported RCTs which assessed a surgical technique used to treat or diagnose head and neck cancer published during or after 2011. The CONSORT 2010 checklist was used to evaluate the reporting quality of these trials.

Results 41 papers were included. The mean CONSORT score was 16.5/25 (66\% adherence) and the scores ranged from 7.5 (30\%) to 25. The most common omissions were full trial protocol (found in 14.6\%), participant recruitment method (22\%) and effect size with a precision estimate for all outcome measures (29.3\%). The full design and implementation of the randomisation methods were reported in 6 (14.6\%). Papers published in journals which endorsed CONSORT had significantly higher scores $(p=0.02)$ and the journal impact factor was significantly correlated with CONSORT score $(p=0.01)$.

Conclusion We have identified several pieces of information that are underreported in surgical head and neck cancer RCTs. These omissions make understanding and comparing the methodologies and conclusions of RCTs more difficult. The endorsement of CONSORT by journals improved adherence, suggesting that wider adoption of the checklist may improve reporting.
\end{abstract}

Keywords Systematic review $\cdot$ Randomised controlled trials $\cdot$ Reporting quality $\cdot$ CONSORT $\cdot$ Head and neck cancer . Head and neck surgery

\section{Introduction}

Randomised controlled trials (RCTs) are largely considered the gold standard in determining the efficacy of treatments and interventions in the medical field. The processes used in an RCT, such as randomisation and double-blinding, remove sources of bias which are inherent to other trial designs [1, 2]. According to the Oxford Centre for Evidence-based Medicine, RCTs are classified as level $1 \mathrm{~b}$ in the Levels of Evidence [3].

Netanya Aarabi Canagarajah and George James Porter contributed equally to this work.

Timothy Shun Man Chu

T.Chu1@ newcastle.ac.uk

1 Faculty of Medical Sciences, Newcastle University, Framlington Place, Newcastle Upon Tyne NE2 4HH, UK
Unless research is adequately reported, it can potentially waste billions in investment and detrimentally impact research and patient care [4]. While they may not reflect poor methodology, poor reporting can create doubt about the results and conclusions of both RCTs, and the systematic reviews of which they may be a part [5, 6]. Poor reporting is associated with bias in the estimation of intervention effectiveness, and limits the critical appraisal and results interpretation by the reader [4]. Poor reporting has the potential to affect healthcare decisions at all levels, from an individual patient to national public health policies [7].

In the 1990s, significant shortcomings were identified in the reporting quality of RCTs in general medicine and several specialties. For instance, a study conducted by Ah-See et al. [8] assessed 295 otolaryngology papers over a 30-year period (1966-1995) and identified an unsatisfactory overall mean score of 7.3 out of 12 (scoring proforma based on CONSORT and observations from existing ENT literature). 
Similar findings of historical poor reporting are documented in other disciplines as well $[9,10]$. Efforts have been made to bridge this gap in the reporting quality of RCTs with the introduction of various guidelines such as The Consolidated Standards of Reporting Trials (CONSORT) statement [11].

The CONSORT statement was first conceptualised in 1993 after a group of medical journal editors, authors, epidemiologists and clinical trialists acknowledged the growing pile of evidence highlighting the poor reporting quality of RCTs. They developed the CONSORT Statement, comprising a numbered checklist that guides researchers on the various aspects of how RCTs are conducted and exposed flaws and inaccuracies in the reporting process [11]. The statement was published in 1996, revised in 2001 and 2010 to take into consideration new concerns.

However, the success of guidelines such as CONSORT depends entirely on the adherence to them. Agha et al. [9] looked at the compliance of 122 urological surgical RCTs in the period 2000-2003 (post-CONSORT) and obtained an average CONSORT score of 11.2 out of a possible 22 . It is well documented that compliance to the CONSORT statement has been poor across several other disciplines as well [12, 13].

Surgical RCTs present certain unique practical and ethical difficulties, such as difficulties associated with blinding and the ethical concerns associated with "sham/placebo" studies [14]. This could contribute to poor reporting in the field, as illustrated by the median CONSORT(NPT) score of 27/42 in a study of randomised trials in surgery by Nagendran et al., which looked at 54 trials from 2011 [15]. The CONSORT Non-Pharmacological Treatments (CONSORT-NPT) extension was introduced in 2008 with the aim to remediate some of these issues. However, there is contention as to whether prospective RCTs and therefore, the CONSORT checklist, are appropriate methods of quality assurance for surgical trials [16]. Apart from the features mentioned above, surgical trials pose difficulties in patient recruitment, the uniqueness of every procedure and quality control, and the duration and cost needed to reach the primary end point [16].

To our knowledge, there were three published studies that focussed specifically on the reporting of surgical interventions in head and neck surgery. The first two studies restricted their findings to a few selected journals only and included papers from a limited time-frame (2011-2014) $[17,18]$. The third selected papers from 1977 to 2012 and focussed mainly on the different reporting quality of papers written by surgeons versus non-surgeons [19]. There is, therefore, need for a wider and more current analysis of the literature base.

In summary, reporting all the relevant components of an RCT is important to elicit the full value of the study to clinicians. The primary objective of our study was to assess the compliance of the reporting of RCTs in head and neck cancer surgery to the 2010 CONSORT checklist and highlight specific shortcomings in reporting quality. Additionally, we analysed the relation between the 5-Year Impact Factor and CONSORT score, and compared the adherence in journals that did and did not endorse CONSORT.

\section{Methods}

This systematic review was carried out in accordance with the Preferred Reporting Items for Systematic Reviews and Meta-Analyses (PRISMA) guidelines [20].

\section{Literature search}

A structured literature search was conducted using PubMed and Embase via OvidOnline by two authors (NAC and GJP). The search was performed on 18/02/2020. Both databases listed studies up to week six of 2020, from January 1954 for PubMed and January 1988 for Embase.

A full literature search strategy can be found in ESM Appendix A1. In brief, terms such as "Head and Neck Cancer", "Head and Neck Surgery" and "Surgery" were exploded and combined with the Boolean operator 'AND'. The results were then limited to RCTs either via limits native to the search engine or via including "Randomized Controlled Trial" in the search and combining with the Boolean operator 'AND'. Results were then limited to after 2011, as papers published prior to this date were likely to be written before the updated CONSORT statement was published in 2010.

\section{Paper selection}

All articles from the initial search underwent title and abstract screening by GJP, TC and KM. Prospective RCTs where a surgical technique used to treat or diagnose head and neck cancer was the intervention in at least one of the arms were included. Studies were excluded if they were on cadavers or animals, were not reported in English or pertained to non-surgical, peri-operative, cosmetic or ophthalmic interventions not relating to head and neck cancer.

\section{Data extraction}

A data extraction proforma, based on the CONSORT 2010 guideline, was made by TC. Each checklist item and subitem was scored with a simple yes/no answer. Total scores of items 1-25 were then calculated, with a point for each item being granted if all sub-sections were scored as yes or half a point if one out of the two sub-sections were reported. Authors noted down the reasons for deducted points for further analysis. Raters were not blinded. 


\section{Rater training}

The first three papers were read sequentially by both NAC and GJP. Any discrepancies in scoring between the two authors were discussed until a consensus was reached. If agreement could not be reached, a third author (TC) was consulted for the final decision. After this, NAC and GJP produced an agreed guideline to scoring, which they used to score the next paper and once again compared results.

After consensus was reached, the authors (NAC and GJP) each read and scored half of the papers. A further ten papers were scored by both raters to check overall rater agreement after the moderation processes.

\section{Statistical analysis}

Statistical analysis was carried out using SPSS version 26 (IBM, Armonk, NY, USA) and $p<0.05$ was considered statistically significant. Percentage agreement between the two raters was used to identify the impact of the moderation processes as well as identify any major disagreements. Journal impact factor was harvested from Clarivate Analytics [21]. The correlation between journal impact factor and mean adherence to CONSORT was assessed via Spearman's rank correlation coefficient. An independent samples $T$ test was used to compare the differences in CONSORT scores between journals which did and did not endorse CONSORT, as the data met all the relevant assumptions including normality and homogeneity of variance. The same test was used to assess the difference in impact factors between journals which did and did not endorse CONSORT. Where appropriate, $95 \%$ confidence intervals $(\mathrm{CI})$ were also calculated.

\section{Results}

\section{Papers included}

The structured literature search identified 443 articles. After deduplication and title and abstract screening, 51 articles were subjected to full-text assessment, of which 10 were excluded. Forty-one papers that fulfilled the inclusion criteria were included in our analysis (ESM Appendix A2). Figure 1 outlines paper selection via the PRISMA flow diagram.
Fig. 1 A flow chart adapted from the PRISMA guidelines illustrating the paper screening process and the reasons for exclusion during full-text screening [20]

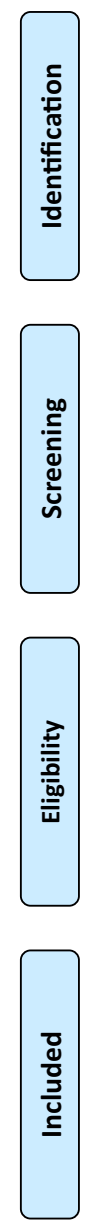




\section{Agreement among parallel readers}

During the first round of scoring by NAC and GJP, the raw percentage agreement was $65.8 \%$. After producing an agreed guideline, the raw percentage agreement increased to $73.0 \%$. After further discussion and revision of the guideline, the agreement between the two raters was $92.01 \%$ in the ten papers scored after the moderation processes.

\section{Sample characteristics}

The 41 included papers were from 26 journals. There were three journals contributing more than 2 papers, these were European Archives of Oto-Rhino-Laryngology (6 papers), Head and Neck-Journal for the Sciences and Specialities of the Head and Neck (4 papers) and Otolaryngology—Head and Neck Surgery (3 papers).

\section{Overall adherence to CONSORT checklist}

Only 1 paper reported all 25 items (100\% adherence) of the CONSORT checklist. The scores ranged from 7.5 (30\% adherence) to 25 (100\%). The 41 papers had a mean score of 16.5 [66\% adherence; $95 \% \mathrm{CI}(60.7-71.3 \%)$ ] and a median score of $16(64 \%)$. Figure 2 shows a bar chart with frequencies of total scores from all papers.

\section{Adherence to individual items of checklist}

The CONSORT checklist items of scientific background, scientific objectives and interpretation of results were present in all 41 papers $(100 \%)$. Items which were poorly reported include identifying personnel responsible for the execution of different aspects of the trial $(9 / 41,22 \%)$ and the estimated effect size and its precision of results (12/41,29.3\%). Table 1 outlines the reporting frequency of each checklist item.

\section{CONSORT Endorsement and CONSORT Score}

Only 9 out of the 26 journals officially endorse the use of CONSORT [22]. An Independent samples $T$-test was carried out to compare scores from journals which endorsed the use of CONSORT and those that did not. This resulted in a significant difference with $t=2.45$ (95\% CI 2.48-29.13), $p=0.02$. Figure 3 shows a box plot comparing CONSORT adherence between these two variables.

\section{Journal impact factor}

The Clarivate Journal Citation Reports provided us with the 2018 5-year impact factor for these journals [21]. At the time of writing, the Journal Citation Reports 2019 data update had been released in late June 2020 but was still subject to modifications. Therefore, we continued to use the 2018 data [23].

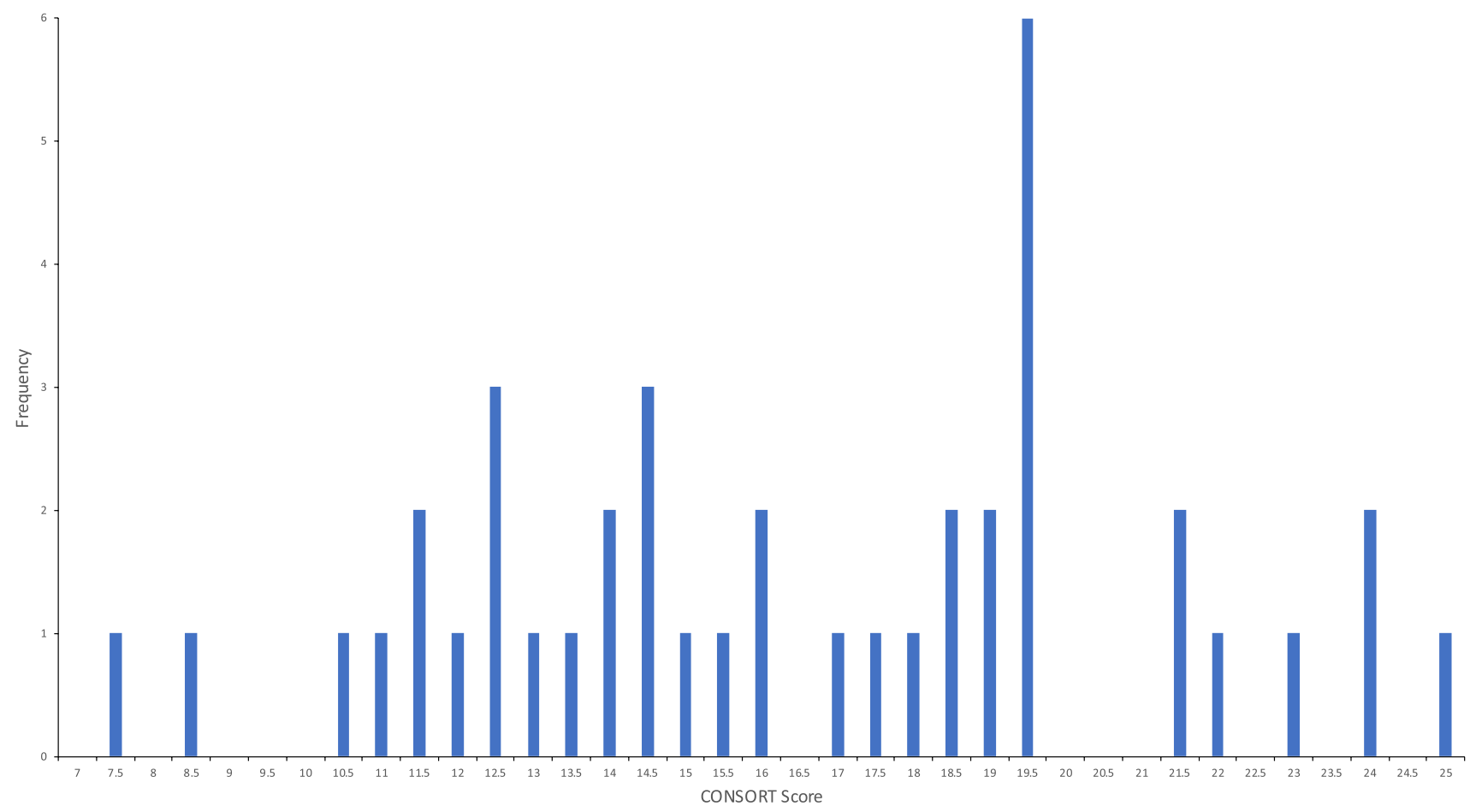

Fig. 2 A bar chart illustrating the frequencies of the total CONSORT scores from all 41 RCTs 
Table 1 The frequency and adherence of RCTs to individual items of CONSORT 2010 checklist, in order of increasing fulfillment

\begin{tabular}{|c|c|c|c|}
\hline Item & Description & Frequency & Adherence $(\%)$ \\
\hline 24 & Where the full trial protocol can be accessed, if available & $6 / 41$ & 14.6 \\
\hline $4 \mathrm{a}$ & Eligibility criteria for participants & $8 / 41$ & 19.5 \\
\hline 10 & $\begin{array}{l}\text { Who generated the random allocation sequence, who enrolled participants, and who assigned participants to } \\
\text { interventions }\end{array}$ & $9 / 41$ & 22.0 \\
\hline $17 \mathrm{a}$ & $\begin{array}{l}\text { For each primary and secondary outcome, results for each group, and the estimated effect size and its preci- } \\
\text { sion (such as } 95 \% \text { confidence interval) }\end{array}$ & $12 / 41$ & 29.3 \\
\hline 9 & $\begin{array}{l}\text { Mechanism used to implement the random allocation sequence (such as sequentially numbered containers), } \\
\text { describing any steps taken to conceal the sequence until interventions were assigned }\end{array}$ & $13 / 41$ & 31.7 \\
\hline $3 \mathrm{a}$ & Description of trial design (such as parallel, factorial) including allocation ratio & $16 / 41$ & 39.0 \\
\hline $8 \mathrm{~b}$ & Type of randomisation; details of any restriction (such as blocking and block size) & $16 / 41$ & 39.0 \\
\hline 25 & Sources of funding and other support (such as supply of drugs), role of funders & $17 / 41$ & 41.5 \\
\hline 23 & Registration number and name of trial registry & $19 / 41$ & 46.3 \\
\hline $14 b$ & Why the trial ended or was stopped & $21 / 41$ & 51.2 \\
\hline $4 \mathrm{~b}$ & Settings and locations where the data were collected & $22 / 41$ & 53.7 \\
\hline $7 \mathrm{a}$ & How sample size was determined & $23 / 41$ & 56.1 \\
\hline $8 \mathrm{a}$ & Method used to generate the random allocation sequence & $24 / 41$ & 58.5 \\
\hline 21 & Generalisability (external validity, applicability) of the trial findings & $24 / 41$ & 58.5 \\
\hline $14 \mathrm{a}$ & Dates defining the periods of recruitment and follow-up & $26 / 41$ & 63.4 \\
\hline 19 & All important harms or unintended effects in each group & $26 / 41$ & 63.4 \\
\hline 16 & $\begin{array}{l}\text { For each group, number of participants (denominator) included in each analysis and whether the analysis was } \\
\text { by original assigned groups }\end{array}$ & $27 / 41$ & 65.9 \\
\hline 1a & Identification as a randomised trial in the title & $28 / 41$ & 68.3 \\
\hline $13 b$ & For each group, losses and exclusions after randomisation, together with reasons & $28 / 41$ & 68.3 \\
\hline 20 & Trial limitations, addressing sources of potential bias, imprecision, and if relevant, multiplicity of analyses & $28 / 41$ & 68.3 \\
\hline $17 b$ & For binary outcomes, presentation of both absolute and relative effect sizes is recommended & $29 / 41$ & 70.7 \\
\hline $11 \mathrm{a}$ & $\begin{array}{l}\text { If done, who was blinded after assignment to interventions (for example, participants, care providers, those } \\
\text { assessing outcomes) and how }\end{array}$ & $32 / 41$ & 78.0 \\
\hline $13 \mathrm{a}$ & $\begin{array}{l}\text { For each group, the numbers of participants who were randomly assigned, received intended treatment, and } \\
\text { were analysed for the primary outcome }\end{array}$ & $33 / 41$ & 80.5 \\
\hline $6 \mathrm{a}$ & $\begin{array}{l}\text { Completely defined pre-specified primary and secondary outcome measures, including how and when they } \\
\text { were assessed }\end{array}$ & $34 / 41$ & 82.9 \\
\hline 15 & A table showing baseline demographic and clinical characteristics for each group & $34 / 41$ & 82.9 \\
\hline $12 \mathrm{~b}$ & Methods for additional analyses, such as subgroup analyses and adjusted analyses & $38 / 41$ & 92.7 \\
\hline 18 & $\begin{array}{l}\text { Results of any other analyses performed, including subgroup analyses and adjusted analyses, distinguishing } \\
\text { pre-specified from exploratory }\end{array}$ & $38 / 41$ & 92.7 \\
\hline $3 b$ & Important changes to methods after trial commencement (such as eligibility criteria), with reasons & $39 / 41$ & 95.1 \\
\hline $11 \mathrm{~b}$ & If relevant, description of the similarity of interventions & $39 / 41$ & 95.1 \\
\hline $12 \mathrm{a}$ & Statistical methods used to compare groups for primary and secondary outcomes & $39 / 41$ & 95.1 \\
\hline $1 \mathrm{~b}$ & Structured summary of trial design, methods, results, and conclusions & $40 / 41$ & 97.6 \\
\hline 5 & $\begin{array}{l}\text { The interventions for each group with sufficient details to allow replication, including how and when they } \\
\text { were actually administered }\end{array}$ & $40 / 41$ & 97.6 \\
\hline $6 b$ & Any changes to trial outcomes after the trial commenced, with reasons & $40 / 41$ & 97.6 \\
\hline $2 \mathrm{a}$ & Scientific background and explanation of rationale & $41 / 41$ & 100 \\
\hline $2 b$ & Specific objectives or hypotheses & $41 / 41$ & 100 \\
\hline $7 b$ & When applicable, explanation of any interim analyses and stopping guidelines & $41 / 41$ & 100 \\
\hline 22 & Interpretation consistent with results, balancing benefits and harms, and considering other relevant evidence & $41 / 41$ & 100 \\
\hline
\end{tabular}

Three journals making up four papers did not have a 5-year impact factor. These were the International Journal of Surgical Oncology (1 paper), Journal of
Laparoendoscopic and Advanced Surgical Techniques (2 papers) and Saudi Journal of Anaesthesia (1 paper). 


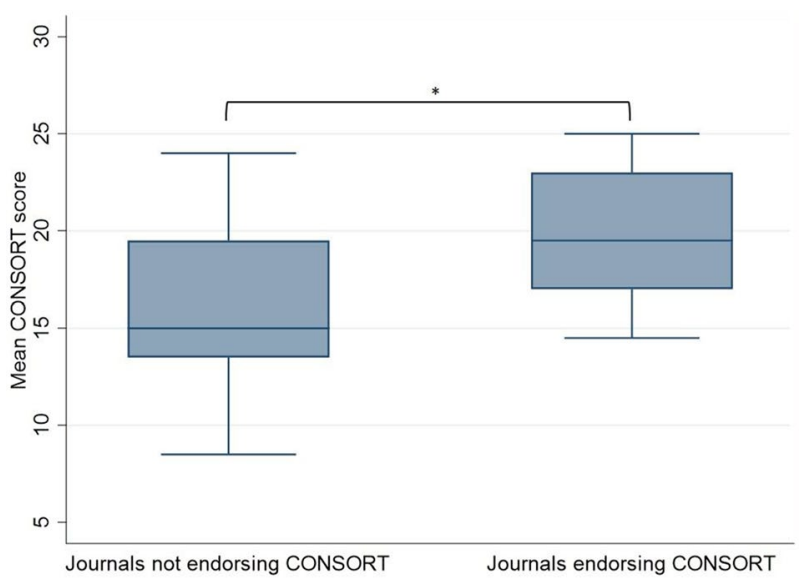

Fig. 3 A box plot to compare adherence to the CONSORT checklist (\%) between journals that endorse the use of CONSORT and those that do not. * Indicates statistical significance at $P<0.05$

There was a statistically significant positive correlation between the journals' 5 -year impact factor and CONSORT score with Spearman $\rho=0.52$ and $p=0.01$. This is illustrated by the scatter plot in Fig. 4 . All journals had a 5 -year impact factor less than 7 except New England Journal of Medicine (NEJM), which had a 5-year impact factor of 70.3 and was an outlier that was excluded from the graph.

The mean impact factor of journals which endorsed CONSORT was 11.4 (6.71 when removing the New England Journal of Medicine, NEJM, as an outlier) while the mean impact factor for journals which did not endorse CONSORT was 2.22. A $t$-test revealed that this was not a statistically significant difference $(p=0.084)$ even when NEJM was included $(p=0.071)$.

\section{Discussion}

With an overall average CONSORT score of $16.5(66 \%$ adherence) from 41 papers, it is clear that there is room for improvement in the reporting quality of surgical head and neck cancer RCTs. Only one paper reported all 25 items (100\% adherence) and the lowest-scoring paper had $30 \%$ adherence.

This study has identified certain pieces of information which are routinely under-reported in manuscripts pertaining to RCTs in the field of head and neck cancer surgery, which limits the application of their results in clinical practice. In particular, the design and implementation of randomisation was only reported in $3(7.3 \%)$ of the included studies. This is problematic as information on randomisation is a crucial requirement for reporting randomised controlled trials. This omission prevents readers from fully judging and understanding the reliability of findings. Furthermore, the eligibility criteria and recruitment method for participants was only explained completely in 8 papers (19.5\%). Finally, precision estimates for the effect sizes of interventions were only presented in $12(29.3 \%)$ of the included papers.

Moreover, several pieces of information designed to reduce 'cherry picking' of results are under-reported. These include full trial protocols (reported in 14.6\%), reason for trial cessation (51.2\%), trial registration (46.3\%) and whether analysis was by intention-to-treat (65.9\%). Inclusion of these data helps ensure that all information harvested, and analyses conducted by the trial are reported. Without this information, it is possible for authors to exclude participants or run analyses in such
Fig. 4 A scatter plot to show the 5-year impact factor vs adherence (\%) to CONSORT checklist

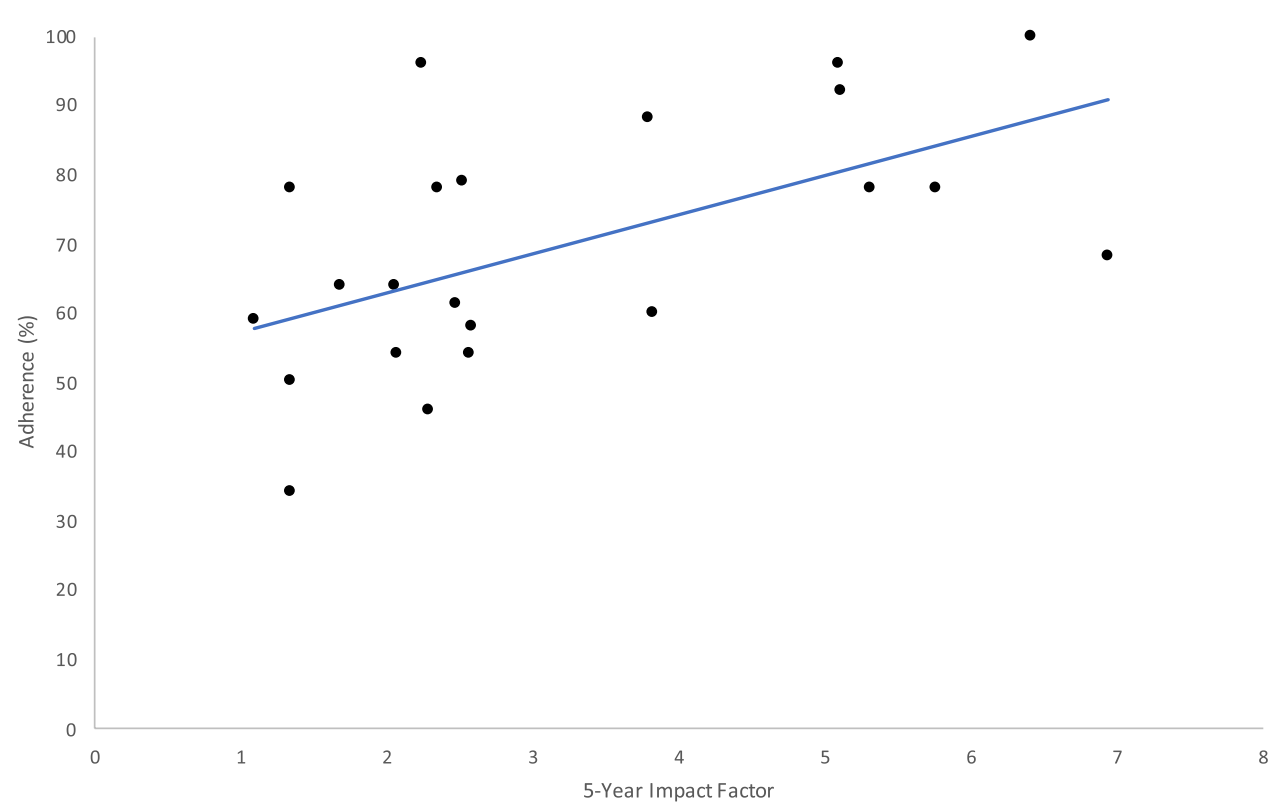


a way to magnify the observed differences between the trial arms. The reason RCTs are seen as a 'gold standard' of investigation is their ability to exclude biases, and the reporting of this information is important in this regard. Similarly, the sources of funding and roles of sponsors in a trial was only reported in $41.5 \%$ of the trials. This is a potential source of bias which should be explicitly stated in the text.

Several items were very well reported. Scientific background and rationale, objectives, consistent and interpretation of results were found in all studies. The use of the CONSORT flow diagram was very prevalent throughout the included studies as well as clear descriptions of the interventions used. However, these items are integral to readers' understanding of the trial and therefore their inclusion is to be expected.

Articles from journals which endorsed the CONSORT guidelines had greater mean CONSORT adherence. This is to be expected but also implies that the reporting of RCTs would improve if more journals required CONSORT adherence. This supports the wider adoption of the CONSORT checklist in medical journals. Articles from high impact factor journals also had better CONSORT adherence. However, journals which endorsed CONSORT had a higher mean impact factor and while this difference was not significant $(p=0.07)$, this likely confounded the relationship.

\section{Comparison with existing literature}

The main omissions identified by this study are not unique to head and neck cancer surgical RCTs. Agha et al. [9] conducted a systematic review of surgical RCTs with a special focus on urological trials in 2007. From their 90 included urological studies, the most frequent omissions were on the topic of randomisation, with no studies reporting how their randomisation sequence was implemented. They also found that only $20 \%$ of trials reported their sources of funding [9]. This is concordant with two reviews of reporting quality in plastic surgery RCTs, which both found that information on randomisation was poorly reported, with one study reporting compliance of $11 \%$ while the other found the information in just $2.4 \%$ of included studies [24, 25]. Equally, a review of 120 surgical RCTs found that none included information on randomisation implementation [26].

Items such as trial registration and full trial protocol have been shown to be poorly reported across a range of surgical specialties $[9,26]$. Several trials included in our study did not report precision estimates for all effect sizes. This was replicated by a systematic review of orthopaedic RCT reporting quality [27]. In summary, much of the information that was routinely omitted in surgical head and neck cancer RCTs has been shown to be routinely omitted in other surgical specialities.
Head and neck cancer surgery was chosen because it is a rapidly evolving field and the evaluation of new techniques forms a cornerstone of evidence-base medicine. Despite this, there are several studies in similar areas which serve as useful comparisons. Huang et al. [17] conducted a systematic review of medical and surgical otolaryngology RCTs from a set of specific journals between 2010 and 2014 . They found that only $6.5 \%$ of included papers described randomisation fully and $32.4 \%$ reported the effect size and precision. Carlton et al. [19] also reviewed the reporting quality of surgical head and neck cancer RCTs. However, their results were from 1977 to 2012, a considerably older sample than ours. They also found that randomisation was poorly reported, with the individual who implemented the randomisation sequence being reported in only $2.6 \%$ of their included papers [19]. In summary, several similar reviews to ours have found comparable results, implying that these omissions are consistently present across the literature base.

Journals which endorsed the use of CONSORT had a higher mean CONSORT adherence. This has been replicated in several studies [28], something which adds weight to the argument that endorsement of CONSORT is a viable method on the part of journals to increase reporting quality of trials.

It should be noted that direct comparisons of CONSORT scores between different studies can be unhelpful as different authors have interpreted CONSORT in different manners. Indeed, Hays et al. [29] stated directly that the CONSORT statement is open to significant interpretation and there is a need to reduce the CONSORT checklist ambiguity to improve adherence. This is also evident in the study by Huang et al. [17] who reported inter-rater reliability between their authors as 0.32 using Cohen's $\kappa$ and observed agreement of 0.87. CONSORT was not designed as a checklist to supply a quality score, so raw scores should be interpreted with caution [7]

Some have also stated that the CONSORT checklist is an inappropriate way of judging the reporting quality of surgical trials, and newer methods may be required to ensure reporting quality [16]. Although this could partly justify the low CONSORT scores seen throughout published literature on the topic, reporting quality appears low with major omissions such as randomisation and funding seen in many studies. Therefore, while valid criticisms can be made in the use of CONSORT to judge reporting quality, these do not invalidate the claim that more work is needed in this area to ensure adequate RCT reporting. In our study, we have used CONSORT to highlight routinely under-reported pieces of information in this literature base.

\section{Limitations of methodology}

CONSORT also has an extension for RCTs concerning non-pharmacological treatments (NPTs). We did not use 
this because many studies included in our review involved both surgical and non-surgical interventions. As the latest CONSORT-NPTs version was published in 2017, limiting results to after this date would considerably limit our sample size. Also, the previous version of the CONSORTNPTs did not include several important items, such as the source of funding.

Our literature search was conducted in English and only English language results were included. Also, while we collected data on whether a journal endorsed CONSORT, we did not record when it endorsed CONSORT in relation to the publication of the included studies. Therefore, it is unclear if the journal endorsed CONSORT at the time of publication. Given that the first iteration of CONSORT was published in 1996, this is unlikely to have made a large impact but is a potential inaccuracy.

Interrater agreement was high in our study and we had several measures to reduce individual biases, such as coscoring of papers both before and after the main body was analysed. Despite this, we cannot completely exclude bias between the two raters.

\section{Conclusion}

We have identified several pieces of information that are routinely underreported in surgical head and neck cancer RCTs. These make the comparison of methodologies of RCTs and the clinical application of trial results difficult due to missing information. In particular, detail on the randomisation processes used as well as the funding sources are key requirements for ensuring that a trial has minimised biases, and the omission of these is especially problematic.

Endorsement of CONSORT by journals improved adherence, suggesting that wider adoption of the checklist may improve reporting. There are several papers assessing the reporting quality of RCTs in various surgical specialties. Many of the findings of such papers are similar with detail on randomisation and full reporting of precision measures for effect sizes being common omissions. Given these reciprocal findings across manuscripts over several years it is possible that more explicit guidance from journals, academic bodies and CONSORT is required to improve reporting in these areas. For example, clinical academic departments, as well as journal editorial offices, could release explicit guidance on how to appropriately design, implement and report randomisation methods as well as the importance of reporting protocols and the roles of funders in the research.

Supplementary Information The online version contains supplementary material available at https://doi.org/10.1007/s00405-021-06694-9.
Acknowledgements We thank the Newcastle University Clinical Academic Office, The Academy of Medical Sciences and the Wellcome Trust for their support of this work through the Newcastle University INSPIRE scheme. The content of this article is solely the responsibility of the authors and does not necessarily represent the official views of these organisations.

Author contributions NAC and GJP: substantial contributions to the conception, design, data acquisition, analysis, interpretation of data for the work, drafting the work and revising it critically for important intellectual content; KM: substantial contributions to the design, data acquisition, interpretation of data for the work, drafting the work and revising it critically for important intellectual content; TC: substantial contributions to the conception, design, data acquisition, analysis, interpretation of data for the work, revising the work critically for important intellectual content.

Funding The authors did not receive support from any organization for the submitted work.

Availability of data and materials Not applicable.

Code availability Not applicable.

\section{Compliance with ethical standards}

Conflict of interest The authors have no financial or proprietary interests in any material discussed in this article.

Ethics approval Not applicable.

Consent to participate Not applicable.

Consent for publication Not applicable.

Open Access This article is licensed under a Creative Commons Attribution 4.0 International License, which permits use, sharing, adaptation, distribution and reproduction in any medium or format, as long as you give appropriate credit to the original author(s) and the source, provide a link to the Creative Commons licence, and indicate if changes were made. The images or other third party material in this article are included in the article's Creative Commons licence, unless indicated otherwise in a credit line to the material. If material is not included in the article's Creative Commons licence and your intended use is not permitted by statutory regulation or exceeds the permitted use, you will need to obtain permission directly from the copyright holder. To view a copy of this licence, visit http://creativecommons.org/licenses/by/4.0/.

\section{References}

1. Akobeng AK (2005) Understanding randomised controlled trials. Arch Dis Child 90:840-844. https://doi.org/10.1136/ adc. 2004.058222

2. Gold standard of evidence: the randomized controlled trial (RCT). https://iancommunity.org/cs/understanding_research/randomized _controlled_trials. Accessed 24 Aug 2020

3. Phillips B, Ball C, Sackett D et al (2009) Oxford Centre for Evidence-based Medicine Levels of Evidence. https://www.cebm. ox.ac.uk/resources/levels-of-evidence/oxford-centre-for-evide 
nce-based-medicine-levels-of-evidence-march-2009. Accessed 24 Aug2020

4. Chan AW, Song F, Vickers A et al (2014) Increasing value and reducing waste: addressing inaccessible research. Lancet 383:257-266. https://doi.org/10.1016/S0140-6736(13)62296-5

5. Soares HP, Daniels S, Kumar A et al (2004) Bad reporting does not mean bad methods for randomised trials: observational study of randomised controlled trials performed by the Radiation Therapy Oncology Group. Br Med J. https://doi.org/10.1136/ bmj.328.7430.22

6. Dechartres A, Trinquart L, Atal I et al (2017) Evolution of poor reporting and inadequate methods over time in 20920 randomised controlled trials included in Cochrane reviews: research on research study. BMJ. https://doi.org/10.1136/bmj.j2490

7. Schulz KF, Altman DC, Moher D (2010) CONSORT 2010 statement: updated guidelines for reporting parallel group randomised trials. Ital J Public Health. https://doi.org/10.4178/epih/e2014029

8. Ah-See KW, Molony NC (1998) A qualitative assessment of randomized controlled trials in otolaryngology. J Laryngol Otol 112:460-463

9. Agha R, Cooper D, Muir G (2005) The reporting quality of randomised controlled trials in surgery: a systematic review. Int $\mathrm{J}$ Surg 5:413-422

10. Kayode Adetugbo PhD, Hywel Williams P (2000) How well are randomized controlled trials reported in the dermatology literature? Arch Dermatol 136:381-385

11. How CONSORT Began. http://www.consort-statement.org/about -consort/history. Accessed 24 Aug 2020

12. Studies on the impact of and adherence to CONSORT. http:// www.consort-statement.org/about-consort/studies-on-the-impac t-of-and-adherence-to-consort. Accessed 25 Aug 2020

13. Gogtay JN (2019) Reporting of randomized controlled trials: will it ever improve? Perspect Clin Res 10:49-50

14. Das AK (2011) Randomised clinical trials in surgery: a look at the ethical and practical issues. Indian J Surg 73(4):245-250. https:// doi.org/10.1007/s12262-011-0307-5

15. Nagendran M, Harding D, Teo W et al (2013) Poor adherence of randomised trials in surgery to CONSORT guidelines for nonpharmacological treatments (NPT): a cross-sectional study. BMJ Open 3:e003898. https://doi.org/10.1136/bmjopen-2013-003898

16. Evrard S, McKelvie-Sebileau P, Van De Velde C et al (2016) What can we learn from oncology surgical trials? Nat Rev Clin Oncol 13:55-62.https://doi.org/10.1038/nrclinonc.2015.176

17. Huang YQ, Traore K, Ibrahim B, et al (2018) Reporting quality of randomized controlled trials in otolaryngology: review of adherence to the CONSORT statement. J Otolaryngol Head Neck Surg 47:34. https://doi.org/10.1186/s40463-018-0277-8
18. Peters JPM, Hooft L, Grolman W, Stegeman I (2015) Assessment of the quality of reporting of randomised controlled trials in otorhinolaryngologic literature-adherence to the CONSORT statement. PLoS ONE. https://doi.org/10.1371/journ al.pone. 0122328

19. Carlton AD, Kocherginsky M, Langerman JA (2014) A systematic review of the quality of randomized controlled trials in head and neck oncology surgery. Laryngoscope 125:146-152

20. Shamseer L, Moher D, Clarke M et al (2015) Preferred reporting items for systematic review and meta-analysis protocols (PRISMA-P) 2015: elaboration and explanation. BMJ 349:g7647. https://doi.org/10.1136/bmj.g7647

21. InCities Journal Citation Reports (2019) Welcome to journal citation reports. https://jcr.clarivate.com/JCRLandingPageAction .action. Accessed 2 Sept 2020

22. Endorsers-Journals and Organizations. http://www.consort-state ment.org/about-consort/endorsers. Accessed 2 Sept 2020

23. Recent Product Updates. http://jcr.help.clarivate.com/Content/ whats-new.htm. Accessed 2 Sept 2020

24. Agha RA, Camm CF, Doganay E, Edison E, Siddiqui MRS, Orgill DP (2014) Randomised controlled trials in plastic surgery: a systematic review of reporting quality. Eur J Plast Surg 37:55-62

25. Gallo L, Wakeham S, Dunn E et al (2020) The reporting quality of randomized controlled trial abstracts in plastic surgery. Aesth Surg J 40:335-341

26. Yu J, Li X, Li Y, Sun X (2017) Quality of reporting in surgical randomized clinical trials. Br J Surg 104:296-303

27. Parsons NR, Hiskens R, Price CL, et al (2011) A systematic survey of the quality of research reporting in general orthopaedic journals. J Bone Jt Surg Ser B 93-B:1154-1159. https://doi. org/10.1302/0301-620X.93B9.27193

28. Shamseer L, Hopewell S, Altman DG et al (2016) Update on the endorsement of CONSORT by high impact factor journals: a survey of journal "Instructions to Authors" in 2014. Trials 17:301. https://doi.org/10.1186/s13063-016-1408-z

29. Hays M, Andrews M, Wilson R et al (2016) Reporting quality of randomised controlled trial abstracts among high-impact general medical journals: a review and analysis. BMJ Open. https://doi. org/10.1136/bmjopen-2016-011082

Publisher's Note Springer Nature remains neutral with regard to jurisdictional claims in published maps and institutional affiliations. 